BULLETIN OF THE

AMERICAN MATHEMATICAL SOCIETY

Volume 81, Number 2, March 1975

\title{
MATRIX DIFFERENTIAL EQUATIONS
}

\author{
BY RAY REDHEFFER
}

Communicated by Alberto Calderón, December 2,1974

Let $\Omega$ be the set of $n$ by $n$ matrices with complex elements, let $R$ denote the set of reals, and let $R_{0}$ denote the interval $\left[0, t_{0}\right)$ for some $t_{0}>0$. We consider the differential relation

$$
0 \in z^{\prime}-f(t, z), \quad t \in R_{0}
$$

where $z(t) \in \Omega$ and $f$ is a function from $R_{0} \times \Omega$ to subsets of $\Omega$. The equation can be interpreted in two senses: Either $z$ is absolutely continuous and the relation holds almost everywhere, or $z$ is continuous and the relation holds except in a countable set.

A function $\phi(t, \rho)$ from $R_{0} \times R$ to $R$ is a uniqueness function if the upper solution of the equation

$$
D^{+} \rho=\phi(t, \rho), \quad t \in R_{0} ; \rho(0)=0
$$

is $\rho=0$. Here $D^{+}$denotes the upper right Dini derivate, though other derivates could be used just as well. The equation (2) is interpreted in the same sense as (1).

We use $|\xi|$ for the Euclidean length of the complex vector $\xi$, so that $|\xi|^{2}=\xi^{*} \xi$. For $z \in \Omega$ a norm and Kamke norm are defined respectively by

$$
\|z\|=\sup |z \xi|, \quad[z]=\sup \operatorname{Re}\left(\xi^{*} z \xi\right), \quad(|\xi|=1) .
$$

We say that $f$ satisfies a uniqueness condition if there exist an $\epsilon>0$ and a uniqueness function $\phi$ such that

$$
x \in f(t, u), \quad y \in f(t, v), \quad\|u-v\|<\epsilon
$$

together imply

$$
\left[(u-v)^{*}(x-y)\right] \leqslant\|u-v\| \phi(t,\|u-v\|) .
$$

The hypotheses and conclusions of our theorems hold for $t \in R_{0}$ and, for simplicity, all coefficients in the examples are integrable.

AMS (MOS) subject classifications (1970). Primary 34A1 5, 34 G05.

Copyright $\odot 1975$, American Mathematical Society 
Invariance of the unit ball. If $\|z(0)\| \leqslant 1$ implies $\|z(t)\| \leqslant 1$ for solutions of (1), it is said that the unit ball is invariant. This is the case (and the proof is easy) if there exists $\epsilon>0$ such that the three conditions

$$
1+\epsilon>\|v\|>1, \quad w \in f(t, v), \quad v=z(t)
$$

imply $\left[v^{*} w\right] \leqslant 0$. The following theorem has a uniqueness requirement on $f$, but in other respects the hypothesis is much weaker than that above.

THEOREM 1. Let $f$ satisfy a uniqueness condition. Suppose further that there is some $w \in f(t, v)$ such that the three conditions

$$
\|v\|=1, \quad \eta=v \xi, \quad \xi=v^{*} \eta
$$

together imply $\operatorname{Re}\left(\eta^{*} w \xi\right) \leqslant 0$. Then the unit ball is invariant.

The uniqueness condition is needed only relative to points $v$ on $\|v\|=1$ and $u$ on $1<\|u\|<1+\epsilon$ with $u=z(t)$. For proof, it is sufficient to show that

$$
\liminf _{\alpha \rightarrow 0^{+}} \frac{\|v+\alpha w\|-\left\|_{v}\right\|}{\alpha} \leqslant 0
$$

and the result follows from known invariance theorems [1], [2], [3], [5] as extended in [6]. Because of its relation to (3), the hypothesis $\operatorname{Re}\left(\eta^{*} w \xi\right) \leqslant 0$ under the restrictions of Theorem 1 is referred to as the tangent condition on $\|z\|=1$.

For example, let $z$ satisfy the Riccati equation $z^{\prime}=a+b z+z d+z c z$. Theorem 1 immediately gives a result of Reid [7] and the author, to the effect that the unit ball is invariant if

$$
\operatorname{Re}\left(\eta^{*} a \xi+\eta^{*} b \eta+\xi^{*} d \xi+\xi^{*} c \eta\right) \leqslant 0 \text { for }|\xi|=|\eta|
$$

It is seen, incidentally, that the Riccati equation satisfies the tangent condition for $\|z\|=1$ if it satisfies the tangent condition for $z_{z} z=1$.

Theorem 1 applies with equal ease to equations of higher degree. For instance let

$$
\begin{aligned}
z^{\prime}= & a_{1}+b_{1} z+z d_{1}+z c_{1} z+z z * a_{2} z * z \\
& +z z * b_{2} z+z d_{2} z * z+z z * c_{2} z z * z
\end{aligned}
$$

where the coefficients are integrable. Then invariance of the unit ball follows from (4) with 


$$
a=a_{1}+a_{2}, \quad b=b_{1}+b_{2}, \quad c=c_{1}+c_{2}, \quad d=d_{1}+d_{2} .
$$

Invariance of the order cone. We use the order induced by quadratic forms, so $z \leqslant 0$ holds if and only if $[z] \leqslant 0$. Thus $[z]$ is one of the Kamke norms which generate the order relation [4]. The order cone is said to be invariant if $z(0) \geqslant 0$ implies $z(t) \geqslant 0$ for $t \in R_{0}$.

THEOREM 2. Let $f$ satisfy a uniqueness condition. Suppose further that there is some $w \in f(t, v)$ such that the two conditions

$$
v+v^{*} \geqslant 0, \quad\left(v+v^{*}\right) \xi=0
$$

together imply $\xi^{*}\left(w+w^{*}\right) \xi \geqslant 0$. Then the order cone is invariant.

For proof, note that the functionals generating the order cone are of the form $\phi_{\xi}$, where $\phi_{\xi}(z)=\operatorname{Re}\left(\xi^{*} z \xi\right)$ and $|\xi|=1$. It is possible to show that the quasimonotony condition of Volkmann [8] holds under the hypothesis of Theorem 2; hence by [5] the tangent condition holds on the order cone; and Theorem 2 follows from [1], [2], [3], [5] as extended in [6]. For singlevalued functions Theorem 2 also follows from [4], Theorem 2; note that $[p]=1,[-p]=-1$ where $p$ is the identity matrix.

Here again, $v$ and $w$ are somewhat more restricted than stated in the theorem. In particular if the differential equation is Hermitian, so that the solution satisfies $z=z^{*}$ and $f(z, t)=f(z, t)^{*}$, then the hypothesis is needed only when $v=v^{*}$ and $w=w^{*}$.

As a simple illustration let $z^{\prime} \geqslant b z+z b^{*}+z c z+z c z^{*}$. Then $z(0) \geqslant 0$ implies $z(t) \geqslant 0$; compare Reid [7]. If $T u=u^{\prime}-g(t, u)$ monotony in the sense of Collatz can be deduced by applying Theorem 2 with $f(t, z)=$ $g(t, u+z)-g(t, u)$. For example let $T u=u^{\prime}-u b-b^{*} u-u c u$ where $u^{*} c^{*}=$ $u c$. Then $T u \leqslant T v$ and $u(0) \leqslant v(0)$ implies $u(t) \leqslant v(t)$. Note that the choice $g(t, u)=u^{2}$ is permissible here though specifically excluded in [7, Theorem 6.1]. All these applications of Theorem 2 extend to equations of higher degree and to the comparison of two operators, as in $T_{1} u \leqslant T_{2} v$.

Cayley transforms and periodic solutions. It is not difficult to show that the transformation

$$
w=(z-1)(z+1)^{-1}, \quad z=(1+w)(1-w)^{-1}
$$

effects a formal conversion of either Theorem 1 or 2 into the other. Thus the two theorems can be regarded as being in reality a single one, even though they have their roots in two rather distinct historical traditions. This observa- 
tion gives a unified approach to a substantial and diverse literature.

The Cayley transform also has an interesting bearing on periodic solutions. Let $f(z, t)$ have $t$-period $\omega$. If the unit ball is invariant then, subject to mild continuity conditions, the transformation $z(0) \rightarrow z(\omega)$ has a fixed point in $\|z\| \leqslant 1$, and a periodic solution exists. A slightly sharper hypothesis ensures $\|z(t)\|<1$ for $t>0$ in Theorem 1 , so that the fixed point is also in $\|z\|<1$; this is sometimes desirable for technical reasons. In the case of a Riccati equation the transformation $z(0) \rightarrow z(\omega)$ is a linear fractional transformation, the theory of fixed points is simpler than in the general case, and the argument extends to equations in which the unknowns are operators on a Hilbert space.

This discussion gives existence of a periodic solution under conditions in which the main hypothesis is that of Theorem 1 . However the argument does not apply to Theorem 2 , because the set $z \geqslant 0$ is not compact even in the finite-dimensional case. But by a Cayley transformation $z \rightarrow w$ we can get a periodic solution $w$ from Theorem 1 , and transforming back gives a periodic $z$. Hence, there are theorems asserting existence of a periodic solution in which the main hypothesis is that of Theorem 2 .

\section{REFERENCES}

1. P. Hartman, On invariant sets and on a theorem of Ważewski, Proc. Amer. Math. Soc. 32 (1972), 511-520. MR 45 \#7143.

2. V. Lakshmikantham and G. S. Ladde, On flow invariant sets, Technical Report 28, University of Rhode Island, 1972.

3. M. Nagumo, Über die Lage der Integralkurven gewöhnlicher Differentialgleichungen, Proc. Phys.-Math. Soc. Japan (3) 24 (1942), 551-559. MR 7, 381.

4. R. M. Redheffer, Gewöhnliche Differentialungleichungen mit quasimonotonen Funktionen in normierten linearen Räumen, Arch. Rational Mech. Anal. 52 (1973), 121133.

5. R. M. Redheffer and Walter Wolfgang, Flow-invariant sets and differential inequalities in normed spaces (to appear).

6. - A differential inequality for the distance function in normed linear spaces, Math. Ann. 211 (1974), 299-314.

7. W. T. Reid, Riccati differential equations, Academic Press, New York, 1972. A comprehensive list of additional references is given here. Results related to those of this paper are: p. 83, Theorem 11.2, p. 119, Theorem 4.1, p. 121, Lemma 4.1, p. 122, Theorem 4.3, p. 123, Corollary, p. 129, Lemma 6.1, p. 130, Theorem 6.1.

8. P. Volkmann, Gewöhnliche Differentialungleichungen mit quasimonoton wachsenden Funktionen in topologischen Vektorräumen, Math. Z. 127 (1972), 157164. MR 46 \#7661.

DEPARTMENT OF MATHEMATICS, UNIVERSITY OF CALIFORNIA, LOS ANGELES, CALIFORNIA 90024 